\title{
Efeitos do treinamento de força na capacidade funcional e saúde de idosos: uma revisão integrativa
}

\author{
Effects of strength training on functional capacity and health of the elderly: an integrative review
}

Efectos del entrenamiento de fuerza en la capacidad funcional y la salud de los ancianos: una revisión integradora

\section{Resumo}

Objetivo: Identificar os efeitos do treinamento de força na capacidade funcional e saúde de idosos. Metodologia: Tratase de um estudo de revisão integrativa, que analisou artigos publicados de 2010 a 2020 nas bases de dados Lilacs, SciElo e Pubmed, selecionados na busca online dos descritores exatos e sinônimos em português e em inglês dos termos "treinamento de força", "capacidade física funcional" e "idoso". Foram incluídos artigos com texto completo disponível em meio online, de forma gratuita, nos idiomas português, inglês ou espanhol, publicados de 2010 a 2020 para que obtivesse um contexto atualizado sobre os efeitos do treinamento de força com o público-alvo deste estudo. A amostra final foi composta por 10 artigos. Resultados: Os dados indicaram que o treinamento de força em intensidade moderada ou vigorosa proporciona benefícios aos idosos por meio do próprio ganho de força muscular e melhora da capacidade aeróbia, que diminuem os riscos de quedas, além da redução da pressão arterial e de sintomas depressivos, seja em idosos saudáveis ou com comorbidades. Considerações Finais: Percebeu-se, por meio desta revisão que o treinamento de força melhora a capacidade física funcional de idosos e favorece um envelhecimento saudável, pois reduz o risco de quedas e colabora para o controle de doenças crônicas.

Palavras-chave: Treinamento de força; Capacidade física funcional; Idoso.

\begin{abstract}
Objective: To identify the effects of strength training on the functional capacity and health of the elderly. Methodology: This is an integrative review study, which analyzed articles published from 2010 to 2020 in the Lilacs, SciElo and Pubmed databases, selected in an online search for exact descriptors and synonyms in Portuguese and English for the terms "strength training", "functional physical capacity" and "elderly". Full text articles available online, free of charge, in Portuguese, English or Spanish, published from 2010 to 2020, were included in order to obtain an updated context on the effects of strength training with the target audience of this study. The final sample consisted of 10 articles. Results: The data indicated that strength training at moderate or vigorous intensity provides benefits to the elderly through their own gain in muscle strength and improvement in aerobic capacity, which reduce the risk of falls, in addition to reducing blood pressure and depressive symptoms, whether in healthy elderly or with comorbidities. Final Considerations: Through this review, it was noticed that strength training improves the functional physical capacity of the elderly and favors healthy aging, as it reduces the risk of falls and contributes to the control of chronic diseases.
\end{abstract}

Keywords: Strength training; Physical functional performance; Aged. 


\begin{abstract}
Resumen
Objetivo: Identificar los efectos del entrenamiento de fuerza sobre la capacidad funcional y la salud de las personas mayores. Metodología: Se trata de un estudio de revisión integradora, que analizó artículos publicados entre 2010 y 2020 en las bases de datos Lilacs, SciElo y Pubmed, seleccionados en una búsqueda en línea de descriptores exactos y sinónimos en portugués e inglés para los términos "entrenamiento de fuerza", "capacidad física funcional" y “ancianos". Se incluyeron artículos de texto completo disponibles en línea, de forma gratuita, en portugués, inglés o español, publicados de 2010 a 2020, con el fin de obtener un contexto actualizado sobre los efectos del entrenamiento de fuerza con el público objetivo de este estudio. La muestra final estuvo formada por 10 artículos. Resultados: Los datos indicaron que el entrenamiento de fuerza a intensidad moderada o vigorosa brinda beneficios a los ancianos a través de su propia ganancia de fuerza muscular y mejora de la capacidad aeróbica, que reducen el riesgo de caídas, además de disminuir la presión arterial y los síntomas depresivos, ya sea en ancianos sanos o con comorbilidades. Consideraciones finales: A través de esta revisión, se notó que el entrenamiento de fuerza mejora la capacidad física funcional de las personas mayores y favorece el envejecimiento saludable, ya que reduce el riesgo de caídas y contribuye al control de enfermedades crónicas.
\end{abstract}

Palabras clave: Entrenamiento de fuerza; Capacidad física funcional; Anciano.

\title{
1. Introdução
}

A estrutura demográfica da população global vem se modificando, especialmente pelo aumento no número de indivíduos idosos. Estimativas apontam que até 2050 a população mundial com 60 anos ou mais chegará a dois bilhões e há uma preocupação quanto à capacidade dessas pessoas realizarem as atividades do dia a dia de forma satisfatória, já que nem sempre o envelhecimento está atrelado a um estilo de vida saudável (World Health Organization, 2017).

As limitações decorrentes do processo de envelhecimento podem ser agravadas quando o indivíduo adota um estilo de vida com pouca ou nenhuma atividade física habitual (Ferreira, Maciel, Costa, Silva, \& Moreira, 2012), o que contribui para o aparecimento de doenças crônicas (Miranda, Mendes, \& Silva, 2016). Além disso, a redução das capacidades físicas funcionais, como por exemplo, a diminuição da capacidade aeróbica e da potência anaeróbica, representam barreiras para a realização das atividades da vida diária, tornando estes indivíduos mais dependentes e com risco de quedas mais evidentes (Oliveira et al., 2019).

Dentre as alterações fisiológicas mais significativas que ocorrem no processo de envelhecimento, encontra-se a redução da massa magra, caracterizada pela sarcopenia, condição que contribui para a redução da força e da potência anaeróbica (Pícoli, Figueiredo, \& Patrizzi, 2011; Leite et al., 2012). As consequências dessa perda afetam a vida diária da população idosa, que depende da força para realizar atividades cotidianas como sentar e levantar, subir ou descer escadas, carregar compras e demais objetos de uso pessoal. Além de tudo isso, a força se associa com a potência anaeróbica e sua redução aumenta o risco de quedas e de mortes, fator que pode ser diminuído com a prática de exercícios físicos (Pícoli, Figueiredo, \& Patrizzi, 2011).

Nesse sentido, o treinamento de força está entre os exercícios mais recomendados para o público idoso, pois independentemente de outras capacidades físicas, o aumento do tônus muscular pode colaborar para a efetiva realização das atividades da vida diária e para a diminuição da mortalidade por todas as causas (Chodzko-Zakjo et al., 2009). Ademais, acreditase que o treinamento de força pode proporcionar benfeitorias relacionadas à saúde que afetam diretamente na qualidade de vida desta população, tornando-os menos dependentes.

Diante do cenário apresentado, este estudo foi realizado com o objetivo de identificar os efeitos do treinamento de força na capacidade funcional e saúde de idosos com a perspectiva de promover a ampliação do conhecimento relacionado à qualidade de vida desta população.

\section{Metodologia}

Este estudo se caracteriza como uma revisão integrativa da literatura, a qual permite uma sintetização dos principais resultados em relação a determinado tema, seguindo as recomendações de Whittemore e Knafl (2005) para a realização de uma 
pesquisa dessa natureza e de acordo com os critérios do Preferred Reporting Items for Systematic Reviews and Meta-Analyses (PRISMA Statement) (Moher, Liberati, Tetzlaff, Altman, \& The PRISMA Group, 2009).

A estratégia PICO ( $\mathrm{P}$ - population; $\mathrm{I}$ - intervention; $\mathrm{C}$ - comparison; $\mathrm{O}$ - outcomes) foi utilizada para elaborar a questão norteadora da pesquisa: "Quais são os efeitos do treinamento de força na capacidade funcional e saúde de idosos?”.

A busca na literatura foi realizada por dois revisores independentes e às cegas, sendo consultado um terceiro revisor em caso de discordância entre os meses de setembro e outubro de 2020 nas seguintes bases de dados eletrônicas: Literatura Latinoamericana e do Caribe (Lilacs) via Biblioteca Virtual em Saúde (BVS), Scientific Eletronic Library Online (SciELO) e Medline via PubMed.

Os descritores controlados e sinônimos foram selecionados através dos Descritores em Ciências da Saúde (DeCS) e do Medical Subject Headings (MeSH), combinados por meio do operador booleano AND da seguinte maneira: "strenght training" AND "physical functional performance" AND "elderly" no idioma inglês, e "treinamento de força" AND "capacidade física funcional" AND "idoso" no idioma português de acordo com cada base eletrônica de dados.

Foram estabelecidos os seguintes critérios de elegibilidade para a seleção dos artigos, ordenados de acordo com a estratégia PICO: estudos com a população idosa (> 60 anos) com ou sem comorbidades (population); aplicação de sessões do treinamento de força (intervention); comparação com um ou mais grupos: exercícios físicos aeróbicos, de equilíbrio, alongamento, sem atividade física ou manutenção da rotina habitual; efeitos na capacidade física funcional ou variáveis relacionadas à saúde (outcomes).

Além disso, foram incluídos artigos com texto completo disponível em meio online, de forma gratuita, nos idiomas português, inglês ou espanhol, publicados de 2010 a 2020 para que obtivesse um contexto atualizado sobre os efeitos do treinamento de força com o público-alvo deste estudo, sendo excluídos os artigos publicados fora do recorte temporal estabelecido, estudos duplicados, revisões de literatura e pesquisas fora da temática.

Ainda nesta etapa foi realizada a pré-seleção dos artigos com a leitura do título, resumo e palavras-chave, sendo excluídos os artigos repetidos. Então, os artigos pré-selecionados foram lidos na íntegra e, destes, apenas os que cumpriam todos os critérios de inclusão foram utilizados na amostra final deste estudo.

Para avaliar os dados extraídos foi elaborado um instrumento de coleta de dados contendo as seguintes variáveis: base de dados; autor(es) e ano de publicação; tipo de estudo, população e nível de evidência; intervenção realizada; objetivo do estudo e; principais resultados. Não foram utilizados instrumentos para avaliar a qualidade metodológica dos estudos incluídos nesta revisão, entretanto, a seleção dos artigos buscou incluir pesquisas com delineamento experimental e quase-experimentais, além de ensaios clínicos randomizados para que se mantivesse um nível de evidência entre 2 e 3 numa escala de 1 a 6 , sendo o nível 1 considerado uma melhor classificação hierárquica (Souza, Silva, \& Carvalho, 2010).

Para analisar os dados, realizou-se uma categorização dos resultados, permitindo melhor exploração das variáveis analisadas na obtenção de respostas para o problema de pesquisa levantado. As categorias estabelecidas para as análises foram: Benefícios do treinamento de força para a população idosa; Capacidade física funcional X treinamento de força.

Os dados oriundos da busca na literatura foram organizados em um Quadro, possibilitando a análise comparativa das informações e o cotejo com o conhecimento teórico relacionado ao tema.

\section{Resultados}

As buscas nas bases de dados resultaram no total de 210 artigos, dos quais 80 foram localizados na base Lilacs, 16 na SciELO e 114 na PubMed. Após a aplicação dos critérios de inclusão, foram descartados 159 artigos, em seguida, suprimidos 26 estudos com a realização da pré-seleção (leitura do título, resumo e palavras-chave) e, por fim, quatro artigos foram retirados 
por estarem repetidos nas bases de dados. Dos 21 artigos selecionados para serem lidos na íntegra, foram excluídos 11 estudos que não abordavam exatamente a questão temática pretendida neste estudo. Com isso, restaram 10 artigos que compuseram a amostra final desta pesquisa, dos quais quatro foram oriundos da base Lilacs e seis da PubMed (Figura 1).

Figura 1 - Fluxograma da etapa de seleção dos artigos, conforme recomendado pelo Grupo PRISMA.
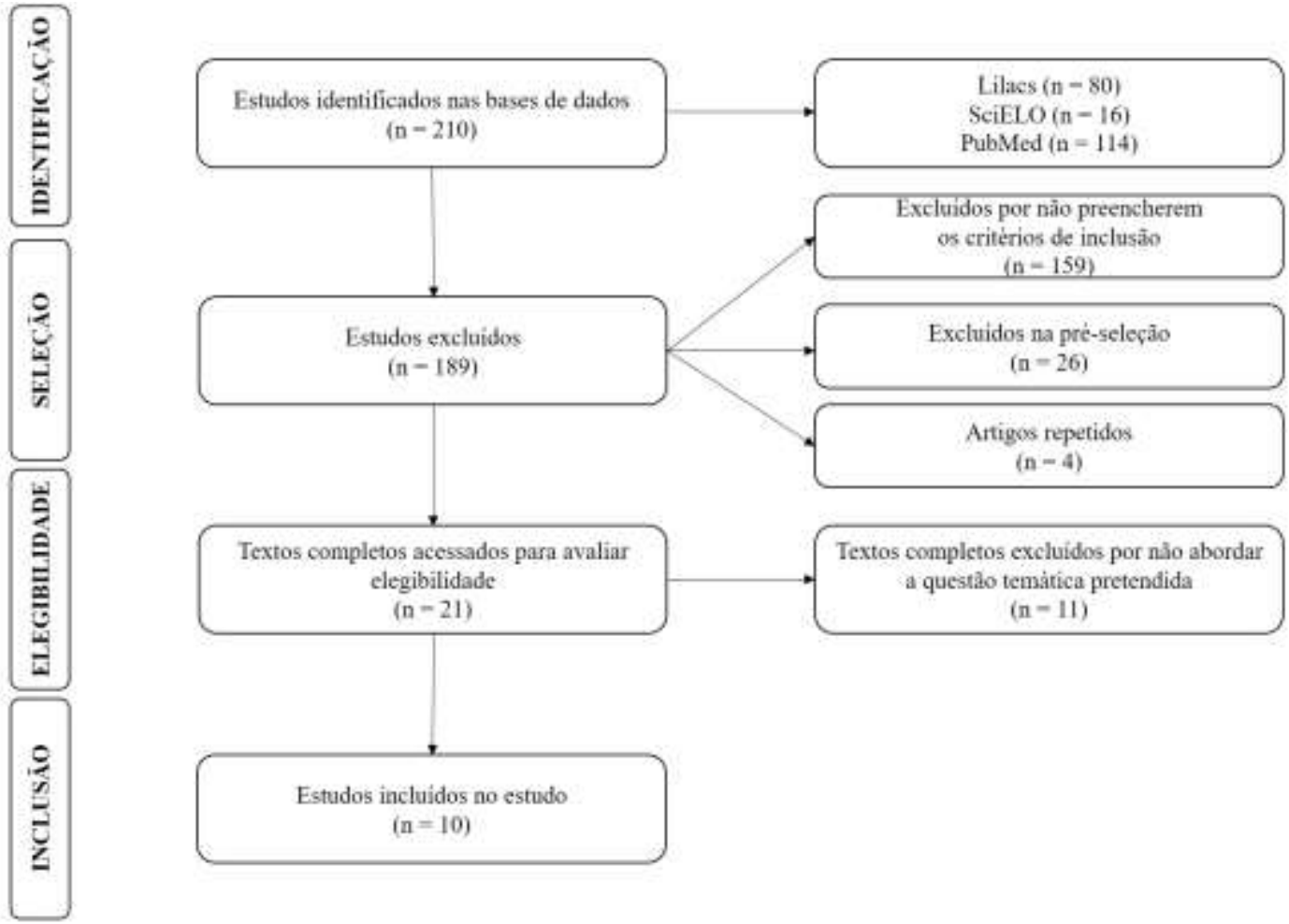

Fonte: Autores (2021).

Os 10 artigos que compuseram a amostra final deste estudo permitiram um vasto recorte temporal das publicações da última década, sendo encontrado pelo menos um artigo de 2012 até 2019. Em relação ao nível de evidência, a maioria (90\%) dos artigos corresponderam ao nível 2, fato que possibilitou uma boa evidência em relação à aplicação do treinamento de força com a população idosa (Quadro 1). 
Quadro 1 - Avaliação dos estudos selecionados de acordo com a ordem cronológica de publicação.

\begin{tabular}{|c|c|c|c|c|c|}
\hline 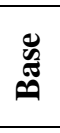 & $\begin{array}{c}\text { Autor(es) e } \\
\text { ano }\end{array}$ & $\begin{array}{c}\text { Tipo de estudo, } \\
\text { população e nível } \\
\text { de evidência }\end{array}$ & Intervenção & Objetivo & Resultados \\
\hline$\frac{\mathscr{U}}{\stackrel{0}{3}}$ & $\begin{array}{l}\text { Moraes et al. } \\
\quad(2012)\end{array}$ & \begin{tabular}{|c|}
$\begin{array}{c}\text { Estudo quase- } \\
\text { experimental não- } \\
\text { controlado. } \\
\\
36 \text { idosos com } \\
\text { hipertensão arterial. } \\
\text { Nível } 3 .\end{array}$ \\
\end{tabular} & $\begin{array}{c}\text { Treinamento de força } \\
\text { durante } 12 \text { semanas, } \\
\text { duas vezes por } \\
\text { semana, duração de } 60 \\
\text { minutos e intensidade } \\
\text { moderada. }\end{array}$ & $\begin{array}{l}\text { Avaliar os efeitos de } \\
\text { um programa de } \\
\text { exercícios físicos nos } \\
\text { níveis pressóricos, na } \\
\text { aptidão física e na } \\
\text { capacidade funcional. }\end{array}$ & $\begin{array}{l}\text { Redução da pressão arterial } \\
\text { sistólica e diastólica. } \\
\text { Melhora da força muscular, } \\
\text { equilíbrio estático, capacidade } \\
\text { aeróbica e da capacidade } \\
\text { funcional. }\end{array}$ \\
\hline$\frac{\tilde{0}}{\pi}$ & $\begin{array}{l}\text { Moura et al. } \\
\text { (2012) }\end{array}$ & 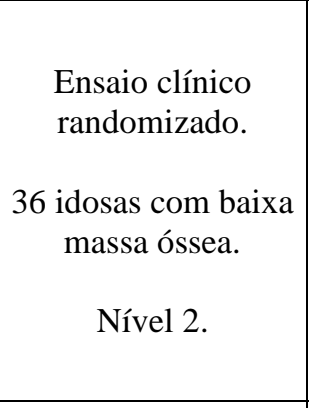 & $\begin{array}{c}30 \text { sessões de } \\
\text { fortalecimento } \\
\text { muscular e mobilidade } \\
\text { funcional e } 30 \text { sessões } \\
\text { de alongamento e } \\
\text { mobilidade articular, } \\
\text { duas vezes por semana } \\
\text { com duração de } 50 \\
\text { minutos. }\end{array}$ & $\begin{array}{l}\text { Avaliar os efeitos de } \\
\text { programas de } \\
\text { exercícios resistidos, } \\
\text { de equilíbrio e de } \\
\text { alongamentos sobre a } \\
\text { mobilidade funcional. }\end{array}$ & $\begin{array}{l}\text { Melhora nos parâmetros } \\
\text { neuromusculares. } \\
\text { Redução do tempo de } \\
\text { realização do teste de sentar- } \\
\text { levantar e do teste de } \\
\text { caminhada. } \\
\text { Aumento do alcance no teste } \\
\text { de alcance funcional. }\end{array}$ \\
\hline 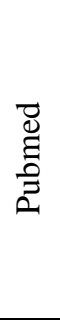 & $\begin{array}{c}\text { Gennuso, } \\
\text { Zalewski, } \\
\text { Cashin e Strath } \\
(2013)\end{array}$ & \begin{tabular}{|c|} 
Estudo \\
experimental. \\
25 idosos com \\
capacidades físicas \\
reduzidas. \\
Nível 2. \\
\end{tabular} & $\begin{array}{l}\text { Treinamento resistido } \\
\text { durante } 8 \text { semanas, } \\
\text { duas vezes por } \\
\text { semana, com } \\
\text { intensidade moderada. }\end{array}$ & $\begin{array}{l}\text { Examinar a eficácia de } \\
\text { duas diretrizes de } \\
\text { treinamento de } \\
\text { resistência para } \\
\text { melhorar a função } \\
\text { física e a classificação } \\
\text { funcional. }\end{array}$ & $\begin{array}{c}\text {-Melhora significativa da } \\
\text { função física, da capacidade } \\
\text { física e da força muscular com } \\
\text { a aplicação das diretrizes. }\end{array}$ \\
\hline 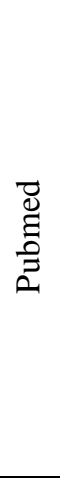 & $\begin{array}{l}\text { Roma et al. } \\
\text { (2013) }\end{array}$ & $\begin{array}{l}\text { Ensaio clínico } \\
\text { randomizado. } \\
96 \text { idosos } \\
\text { sedentários. } \\
\text { Nível } 2 .\end{array}$ & $\begin{array}{c}\text { Treinamento resistido } \\
\text { por } 12 \text { meses, duas } \\
\text { vezes por semana, } \\
\text { duração de } 1 \text { hora e } \\
\text { treinamento aeróbio } \\
\text { (caminhada) por } 12 \\
\text { meses, duas vezes por } \\
\text { semana, duração de } 30 \\
\text { minutos. }\end{array}$ & $\begin{array}{l}\text { Comparar os efeitos } \\
\text { das atividades físicas } \\
\text { resistida e aeróbia } \\
\text { sobre a aptidão física e } \\
\text { funcionalidade de } \\
\text { idosos. }\end{array}$ & $\begin{array}{c}\text { Ambas as atividades } \\
\text { melhoraram a aptidão física. } \\
\text { O treinamento resistido } \\
\text { proporcionou melhora no teste } \\
\text { de sentar/levantar, no } \\
\text { equilíbrio e na flexibilidade. } \\
\\
\text { O treinamento aeróbio } \\
\text { influenciou positivamente na } \\
\text { velocidade, no equilíbrio e na } \\
\text { resistência cardiorrespiratória. }\end{array}$ \\
\hline 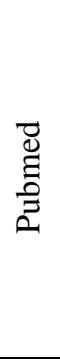 & $\begin{array}{l}\text { Abrahin et al. } \\
\quad(2014)\end{array}$ & $\begin{array}{c}\text { Estudo } \\
\text { experimental. } \\
\\
19 \text { idosas com } \\
\text { experiência em } \\
\text { treinamento } \\
\text { resistido. } \\
\text { Nível 2. }\end{array}$ & $\begin{array}{c}\text { Treinamento resistido } \\
\text { por } 12 \text { semanas, duas } \\
\text { vezes por semana com } \\
\text { intensidade de } 60 \text { a } \\
80 \% \text { de } 10 \mathrm{RM} \\
\text { executando } 1 \text { série e } 3 \\
\text { séries dos exercícios. }\end{array}$ & $\begin{array}{l}\text { Comparar os efeitos do } \\
\text { volume do treinamento } \\
\text { resistido na pressão } \\
\text { inspiratória máxima } \\
\text { (PImáx) e pressão } \\
\text { expiratória máxima } \\
\text { (PEmáx) no } \\
\text { desempenho funcional } \\
\text { e força muscular. }\end{array}$ & $\begin{array}{l}\text { Em ambas as intervenções } \\
\text { houve aumento da PEmáx e } \\
\text { da PImáx, melhora no teste de } \\
\text { sentar/levantar e aumento } \\
\text { significativo da força } \\
\text { muscular. }\end{array}$ \\
\hline 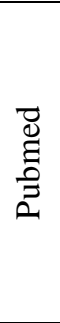 & $\begin{array}{l}\text { Pinto et al. } \\
\text { (2014) }\end{array}$ & $\begin{array}{c}\text { Estudo } \\
\text { experimental. } \\
\\
36 \text { idosas } \\
\text { sedentárias sem } \\
\text { comorbidades. } \\
\text { Nível } 2 .\end{array}$ & $\begin{array}{l}\text { Treinamento de força } \\
\text { por } 6 \text { semanas, duas } \\
\text { vezes por semana, } \\
\text { volume linear e } \\
\text { progressivo, com } \\
\text { séries realizadas até a } \\
\text { falha concêntrica. }\end{array}$ & $\begin{array}{l}\text { Investigar as alterações } \\
\text { na qualidade muscular } \\
\text { do extensor do joelho e } \\
\text { investigar a associação } \\
\text { entre a qualidade } \\
\text { muscular e capacidade } \\
\text { funcional com o } \\
\text { treinamento de força. }\end{array}$ & $\begin{array}{l}\text { Aumento da força muscular, } \\
\text { das medidas de espessura } \\
\text { muscular do quadríceps } \\
\text { femoral, da qualidade } \\
\text { muscular e melhora na } \\
\text { capacidade funcional. }\end{array}$ \\
\hline
\end{tabular}




\begin{tabular}{|c|c|c|c|c|c|}
\hline$\frac{\tilde{U}}{\stackrel{0}{7}}$ & $\begin{array}{c}\text { Brunonni, } \\
\text { Schuch, Dias, } \\
\text { Kruel e } \\
\text { Tiggeman } \\
\text { (2015) }\end{array}$ & $\begin{array}{c}\text { Estudo } \\
\text { experimental. } \\
24 \text { idosas } \\
\text { sedentárias e sem } \\
\text { comorbidades. } \\
\text { Nível } 2 .\end{array}$ & $\begin{array}{l}\text { Treinamento de força } \\
\text { por } 12 \text { semanas, duas } \\
\text { vezes por semana com } \\
\text { cargas progressivas. }\end{array}$ & $\begin{array}{l}\text { Avaliar o efeito do } \\
\text { treinamento de força } \\
\text { com intensidade } \\
\text { prescrita por meio da } \\
\text { percepção de esforço } \\
\text { em idosas na melhora } \\
\text { dos sintomas } \\
\text { depressivos e melhora } \\
\text { da Qualidade de Vida } \\
\text { Relacionada à Saúde. }\end{array}$ & $\begin{array}{l}\text { Aumento significativo da } \\
\text { força máxima, redução nos } \\
\text { sintomas depressivos. } \\
\text { Melhora da qualidade de vida } \\
\text { relacionada à saúde nos } \\
\text { componentes da capacidade } \\
\text { funcional, estado geral de } \\
\text { saúde, vitalidade e saúde } \\
\text { mental. }\end{array}$ \\
\hline 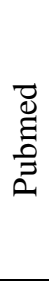 & $\begin{array}{l}\text { Tiggemann et } \\
\text { al. (2016) }\end{array}$ & $\begin{array}{c}\text { Estudo } \\
\text { experimental. } \\
30 \text { idosas saudáveis. } \\
\text { Nível } 2 .\end{array}$ & $\begin{array}{l}\text { Treinamento resistido } \\
\text { tradicional e } \\
\text { treinamento de } \\
\text { potência por } 12 \\
\text { semanas, duas vezes } \\
\text { por semana. }\end{array}$ & $\begin{array}{l}\text { Comparar os efeitos de } \\
12 \text { semanas de } \\
\text { treinamento resistido } \\
\text { tradicional e } \\
\text { treinamento de } \\
\text { potência. }\end{array}$ & $\begin{array}{l}\text { Em ambos os protocolos } \\
\text { houve um aumento } \\
\text { significativo da força } \\
\text { muscular em } 1 \mathrm{RM}, \mathrm{da} \\
\text { potência, do desempenho } \\
\text { físico e da capacidade } \\
\text { funcional. }\end{array}$ \\
\hline$\frac{\tilde{g}}{3}$ & $\begin{array}{l}\text { Mota, Silva, } \\
\text { Teixeira, } \\
\text { Oliveira e } \\
\text { Cardoso } \\
\text { (2018) }\end{array}$ & $\begin{array}{l}\text { Estudo de caso. } \\
\text { Uma idosa com } \\
\text { Síndrome Pós- } \\
\text { Poliomielite. } \\
\text { Nível } 2 .\end{array}$ & $\begin{array}{l}\text { Exercícios aeróbios e } \\
\text { resistidos por } 24 \\
\text { semanas, duas vezes } \\
\text { por semana. }\end{array}$ & $\begin{array}{c}\text { Aplicar um programa } \\
\text { de exercício aeróbio e } \\
\text { de força muscular, e } \\
\text { verificar os resultados } \\
\text { na força muscular e } \\
\text { capacidade funcional } \\
\text { de um indivíduo idoso, } \\
\text { com Síndrome Pós- } \\
\text { Poliomielite. } \\
\end{array}$ & $\begin{array}{l}\text { Aumento da força muscular } \\
\text { de membros inferiores e de } \\
\text { membros superiores. } \\
\text { Melhora da capacidade } \\
\text { funcional e equilíbrio } \\
\text { postural. }\end{array}$ \\
\hline 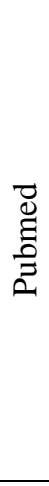 & $\begin{array}{c}\text { Marcos-Pardo } \\
\text { et al. (2019) }\end{array}$ & $\begin{array}{c}\text { Ensaio clínico } \\
\text { randomizado. } \\
45 \text { idosos saudáveis } \\
\text { sem experiência } \\
\text { com o treinamento } \\
\text { de força. } \\
\text { Nível } 2 .\end{array}$ & $\begin{array}{l}\text { Treinamento resistido } \\
\text { por } 12 \text { semanas, três } \\
\text { vezes por semana não } \\
\text { consecutivos, com } \\
\text { intensidade de } 60 \text { a } \\
80 \% \text { de } 1 \mathrm{RM} .\end{array}$ & $\begin{array}{l}\text { Investigar os efeitos de } \\
12 \text { semanas de } \\
\text { treinamento em } \\
\text { circuito de resistência } \\
\text { de intensidade } \\
\text { moderada a alta na } \\
\text { composição corporal, } \\
\text { autonomia funcional, } \\
\text { força muscular e } \\
\text { qualidade de vida. }\end{array}$ & $\begin{array}{l}\text { Aumento da massa corporal } \\
\text { magra e redução da massa } \\
\text { gorda em mulheres e homens; } \\
\text { Melhora na autonomia } \\
\text { funcional e da força muscular } \\
\text { em mulheres e homens; } \\
\text { Não houve diferença } \\
\text { significativa quanto à } \\
\text { qualidade de vida, tanto nas } \\
\text { mulheres quanto nos homens. }\end{array}$ \\
\hline
\end{tabular}

Fonte: Autores (2021).

\section{Discussão}

\subsection{Benefícios do treinamento de força para a população idosa}

Este estudo identificou resultados favoráveis na prática do treinamento de força pela população idosa. Além de contribuir para o aumento da força muscular, como era o esperado, ocorre também melhora na capacidade física funcional e nas condições de saúde de uma forma geral.

No processo de envelhecimento as pessoas tendem a perder massa muscular, fato que influencia diretamente na força muscular e pode levar os idosos a terem dificuldades em realizar movimentos simples e necessários no dia a dia. Porém, essas limitações podem ser atenuadas com a prática do exercício físico (Pillatt, Nielsson, \& Schneider, 2019) e até mesmo melhoradas com a realização do treinamento de força (Pícoli, Figueiredo, \& Patrizzi, 2011; Lixandrão et al., 2016).

Pinto et al. (2014) ao aplicarem o treinamento de força com 36 idosas sedentárias sem comorbidades obtiveram dados positivos com relação ao aumento da força, da qualidade muscular, além de melhorias voltadas à capacidade física funcional. Esses dados mostram a importância da prática do treinamento de força pela população idosa não só para o ganho de massa 
muscular e para aprimoramento da força, mas também como forma de melhorar a autonomia funcional e suas capacidades físicas, o que corroboram com Kalache e Kickbusch (1997) e Kuster, Cruz Razuk e Rinaldi (2021).

Gennuso, Zalewski, Cashin e Strath (2013) aludem como o emprego de diretrizes básicas do American College of Sports Medicine (ACSM) e do American Heart Association (AHA) podem produzir benfeitorias para os idosos. Os autores mostram que os dois protocolos de treinamento de força para idosos com capacidades físicas reduzidas e intensidade moderada foram capazes de melhorar significativamente a função física e força muscular, o que denota que esta prática é segura e produz efeitos favoráveis não somente para pessoas saudáveis (Gennuso, Zalewski, Cashin, \& Strath, 2013).

Da mesma forma, Tiggeman et al. (2016) encontraram resultados semelhantes no que tange ao aumento da força máxima, potência muscular e desempenho funcional em mulheres idosas com a aplicação do treinamento resistido tradicional e com a prática de exercícios de potência (movendo o peso o mais rápido possível), desde que estes programas sejam acompanhados e controlados corretamente.

Há evidências que o treinamento de força tende a aumentar o metabolismo muscular, prevenir doenças arteriais coronarianas e acidente vascular cerebral (Matsudo, Matsudo, \& Barros Neto, 2001; Pereira, Vincensi, Alencar, \& Lima., 2020), bem como as doenças crônicas que têm forte relação com um estilo de vida sedentário e acúmulo de gordura, fato que gera interesse das instituições internacionais (World Health Organization, 2017; 2020) e nacionais (Brasil, 2021) de saúde.

Neste sentido, Moraes et al. (2012) realizaram um estudo quase-experimental com 36 idosos hipertensos e constataram uma redução de 3,6\% da pressão arterial sistólica e de 1,2\% da pressão arterial diastólica, somados a um aumento da capacidade aeróbica, resultados que comprovam os benefícios deste tipo de exercício físico na saúde, assim como Pereira, Vincensi, Alencar e Lima (2020) já destacaram, sendo um treinamento seguro inclusive para pessoas com cardiopatias.

Outros benefícios podem ser notados também na pressão máxima inspiratória e expiratória (Abrahin et al., 2014), redução dos sintomas depressivos, melhora do estado geral de saúde, vitalidade e saúde mental (Brunonni, Schuch, Dias, Kruel, \& Tiggeman, 2015), o que configura um conjunto de ganhos muito além do simples aumento da força muscular.

\subsection{Capacidade física funcional $X$ Treinamento de força}

A redução da capacidade física funcional é um processo natural que acontece ao longo dos anos (Kalache, \& Kickbusch, 1997; Lago, Oliveira, \& Silva, 2021), principalmente na fase do envelhecimento, devido à perda de algumas conexões entre os nervos e músculos que podem ser incapacitantes (Esquenazi, Silva, \& Guimarães, 2014). Todavia, essa perda pode ser reduzida com um programa de treinamento de força que melhore a saúde, desempenho articular e contribua para a melhora de capacidades físicas (Alencar, 2018; Lemos; Guadagnin; \& Mota, 2020).

Sem dúvidas, uma das maiores contribuições do treinamento de força para a população idosa é justamente a melhora das capacidades funcionais, o que devolve a autonomia funcional dessa população e interfere positivamente na qualidade de vida (Grecco, \& Dini, 2017). Essa afirmação fica ainda mais clara no estudo de Marcos-Pardo et al. (2019) e de Brunonni, Schuch, Dias, Kruel e Tiggeman (2015), onde os autores observaram uma evolução da autonomia funcional em testes como o de levantar da posição sentada e de vestir uma camisa (Marcos-Pardo et al., 2019) e uma melhora da qualidade de vida relacionada a saúde (Brunonni, Schuch, Dias, Kruel, e Tiggeman (2015).

O simples ato de subir e descer uma escada, sair do carro, pegar algo do chão ou mesmo mudar de uma posição para outra, às vezes, torna-se muito trabalhoso. O estudo de Roma et al. (2013) que comparou dois modelos de treinamentos distintos na aptidão física e funcional de idosos sedentários, mostrou melhorias nos testes de sentar/levantar e de equilíbrio com o uso do treinamento resistido, resultados que comprovam uma melhora na qualidade de vida e modificação do comportamento no dia a dia na realização de atividades básicas, além de prevenir o risco de quedas (Cunha, \& Pinheiro, 2016). 
Para colaborar com esses resultados, o estudo de caso conduzido por Mota, Silva, Teixeira, Oliveira e Cardoso (2018) com uma idosa de 66 anos, hipertensa, com Síndrome Pós-Poliomielite, dislipidemia e hipotireoidismo, que realizou 24 semanas de exercícios aeróbios e resistidos, revelou que além de melhoras na força muscular e de equilíbrio dinâmico, houve um aumento na distância percorrida, dado também observado por Moura et al. (2012) com a redução do tempo de execução do teste de caminhada de 6 metros por mulheres idosas com baixa massa óssea.

Todas as evidências levantadas neste estudo confirmam que o treinamento de força traz benefícios para a população idosa no que diz respeito à capacidade física funcional, independentemente das condições clínicas. Programas direcionados de treinamento físico como o treinamento de força podem diminuir os efeitos do envelhecimento sobre a função muscular, minimizando as limitações funcionais (Queiroz, \& Munaro, 2012) e melhorando a qualidade de vida e saúde desta população (Wójcicki, \& Mcauley, 2014).

Apesar desta pesquisa não ter utilizado instrumentos para avaliar a qualidade metodológica dos estudos incluídos, o que representa uma limitação a ser considerada na condução de outras revisões, os artigos incorporados à esta revisão seguem um bom nível de evidência, o que contribui para comprovar os efeitos positivos da realização do treinamento de força pela população idosa no que tange à capacidade física funcional e condições de saúde.

\section{Considerações Finais}

Todos os estudos são unânimes quanto aos benefícios que o treinamento de força pode trazer para os idosos, muito além do simples ganho de massa magra ou força muscular, mas também para garantir uma velhice mais segura, prazerosa, independente e com uma melhor qualidade de vida.

Percebeu-se, por meio desta revisão, que o treinamento de força atua fortemente na promoção de um envelhecimento saudável, pois promove melhorias na capacidade física funcional da população idosa, favorece diretamente a redução do risco de quedas e previne doenças crônicas.

Ainda assim, mesmo com a quantidade de evidências já produzidas, outros estudos ainda devem ser desenvolvidos, a fim de identificar as correlações específicas entre o treinamento de força e as capacidades funcionais, para a elaboração de programas de treinamento cada vez mais específicos e seguros para essa população.

\section{Referências}

Abrahin, O., Rodrigues, R. P., Nascimento, V. C., Silva-Grigoletto, M., Sousa, E. C., \& Marçal, A. C. (2014). Single-and multiple-set resistance training improves skeletal and respiratory muscle strength in elderly women. Clinical Interventions in Aging, 9 (4), 1775-1782. https://dx.doi.org/10.2147\%2FCIA.S68529

Alencar, G. P. (2018). Relevância do treinamento de força e suas variáveis nos mais diversos objetivos. FIEP Bulletin, 88 (1), $341-344$. http://www.fiepbulletin.net/index.php/fiepbulletin/article/view/6010

Brasil. Ministério da Saúde. Secretaria de Atenção Primária à Saúde. Departamento de Promoção da Saúde. (2021). Guia de Atividade Física para a População Brasileira. Brasília: Ministério da Saúde. http://bvsms.saude.gov.br/bvs/publicacoes/guia_atividade_fisica_populacao_brasileira.pdf

Brunonni, L., Schuch, F. B., Dias, C. P., Kruel, L. F. M., \& Tiggeman, C. L. (2015). Treinamento de força diminui os sintomas depressivos e melhora a qualidade de vida relacionada a saúde em idosas. Revista Brasileira de Educação Física e Esporte, 29 (2), 189-196. https://doi.org/10.1590/1807-55092015000200189

Chodzko-Zajko, W. J., Proctor, D. N., Fiatarone Singh, M. A., Minson, C. T., Nigg, C. R., Salem, G. J., \& Skinner, J. S. (2009). Exercise and physical activity for older adults. Medicine \& Science in Sports \& Exercise, 41 (7), 1510-1530. https://dx.doi.org/10.1249/MSS.0b013e3181a0c95c

Cunha, P., \& Pinheiro, L. C. (2016). O papel do exercício físico na prevenção das quedas nos idosos: uma revisão baseada na evidência. Revista Portuguesa de Medicina Geral e Familiar, 32 (2), 96-100. Dhttps://www.rpmgf.pt/ojs/index.php/rpmgf/article/view/11732

Esquenazi, D., Ssilva, S. R. B., \& Guimarães, M. A. M. (2014). Aspectos fisiopatológicos do envelhecimento humano e quedas em idosos. Revista HUPE, 13 (2), 11-20. https://doi.org/10.12957/rhupe.2014.10124

Ferreira, O. G. L., Maciel, S. C., Costa, S. M. G., Silva, A. O., \& Moreira, M. A. S. P. (2012). Envelhecimento ativo e sua relação com a independência funcional. Texto e Contexto Enfermagem, 21 (3), 513-518. https://dx.doi.org/10.1590/S0104-07072012000300004 
Gennuso, K. P., Zalewski, K., Cashin, S. E., \& Strath, S. J. (2013). Resistance training congruent with minimal guidelines improves function in older adults: a pilot study. Journal of Physical Activity and Health, 10 (6), 769-776. https://doi.org/10.1123/jpah.10.6.769

Grecco, M. V., \& Dini, R. J. (2017). Como treinar força com segurança na terceira idade. Revista Brasileira de Fisiologia do Exercício, 16 (2), 142-150. http://dx.doi.org/10.33233/rbfe.v16i2.973

Kalache, A., \& Kickbusch, I. (1997). A global strategy for healthy ageing. World Health, 50 (4), 4-5. https://apps.who.int/iris/handle/10665/330616

Kuster, L. M., Cruz, J. L., Razuk, M., \& Rinaldi, N. M. (2021). Benefícios do treinamento de forçanos componentes da capacidade funcional em idosos: uma revisão narrativa. Brazilian Journal of Development, 7 (1), 9851-9867. DOI https://doi.org/10.34117/bjdv7n1-668

Lago, V. M., Oliveira, A. P. S., \& Silva, T. S. (2021). Envelhecimento e mitocôndrias: revisão sistemática de literatura. Mosaicum, s/v (33), 67-84. https://doi.org/10.26893/rm.v33i33.472

Leite, L. E. A., Resende, T. L., Nogueira, G. M., Cruz, I. B. M., Schneider, R. H., \& Gottlieb, M. G. V. (2012). Envelhecimento, estresse oxidativo e sarcopenia: uma abordagem sistêmica. Revista Brasileira de Geriatria e Gerontologia, 15 (2), 365-380. https://doi.org/10.1590/S1809-98232012000200018

Lemos, E. C. W. M., Guadagnin, E. C., \& Mota, C. B. (2020). Influência do treinamento de força e de multicomponentes na funcionalidade de idosos: revisão sistemática e metanálise. Revista Brasileira de Cineantropometria e Desempenho Humano, 22 (e60707). https://doi.org/10.1590/1980-0037.2020v22e6070

Lixandrão, M. E., Damas, F., Chacon-Mikahil, M. P. T., Cavaglieri, C. R., Ugrinowitsh, C., Bottaro, M., Vechin, F. C., Conceição, M. S., Berton, R., \& Libardi, C. A. (2016). Time course of resistance training-induced muscle hypertrophy in the elderly. Journal of Strength \& Conditioning Research, 30 (1), 159-163. https://doi.org/10.1519/jsc.0000000000001019

Matsudo, S. M., Matsudo, V. K. R., \& Barros Neto, T. L. B. (2001). Atividade física e envelhecimento: aspectos epidemiológicos. Revista Brasileira de Medicina do Esporte, 7 (1), 2-13. https://doi.org/10.1590/S1517-86922001000100002

Marcos-Pardo, P. J., Orquin-Castrillón, F. J., Gea-García, G. M., Menayo-Antúnez, R., González-Gálvez, N., Vale, R. G. S., \& Martínez-Rodríguez, A. (2019). Effects of a moderate-to-high intensity resistance circuit training on fat mass, functional capacity, muscular strength, and quality of life in elderly: a randomized controlled trial. Scientific Reports, 9 (1), 1-12. https://doi.org/10.1038/s41598-019-44329-6

Miranda, G. M. D., Mendes, A. C. G., \& Silva, A. L. A. (2016). O envelhecimento populacional brasileiro: desafios e consequências sociais atuais e futuras. Revista Brasileira de Geriatria e Gerontologia, 19 (3), 507-519. https://doi.org/10.1590/1809-98232016019.150140

Moher D, Liberati A, Tetzlaff J, Altman DG, The PRISMA Group. (2009). Preferred reporting items for systematic reviews and meta-analyses: the PRISMA Statement. PLoS Medicine, 6 (7), 1-6. https://doi.org/10.1371/journal.pmed.1000097

Moraes, W. M., Souza, P. R. M., Pinheiro, M. H. N., Irigoyen, M. C., Medeiros, A., \& Koike, M. K. (2012). Programa de exercícios físicos baseado em frequência semanal mínima: efeitos na pressão arterial e aptidão física em idosos hipertensos. Revista Brasileira de Fisioterapia, 16 (2), 114-121. https://doi.org/10.1590/S1413-35552012005000013

Mota, C. G., Silva, S. C. O., Teixeira, B. V., Oliveira, F. M., \& Cardoso, C. V. (2018). Resultados de um programa de exercício físico combinado na força muscular e capacidade funcional de um indivíduo com síndrome Pós-Poliomielite: relato de caso. Acta Fisiátrica, 25 (3), 155-157. https://doi.org/10.11606/issn.2317-0190.v25i3a162646

Moura, M. S., Pedrosa, M. A. C., Costa, E. L., Bastos Filho, P. S. C., Sayão, L. B., \& Sousa, T. S. (2012). Efeitos de exercícios resistidos, de equilíbrio e alongamentos sobre a mobilidade funcional de idosas com baixa massa óssea. Revista Brasileira de Atividade Física \& Saúde, 17 (6), 474-484. https://doi.org/10.12820/rbafs.v.17n6p474-484

Oliveira, D. V., Franco, M. F., Yamashita, F. C., Nascimento, M. A., Freire, G. L. M., Nascimento Júnior, J. R. A., Oliveira, R. V., \& Bertoloni, S. M. M. G. (2019). Comparação da funcionalidade, risco de quedas e medo de cair em idoso em razão do perfil de prática de atividade física. Acta Fisiátrica, 26(4), 176180. https://doi.org/10.11606/issn.2317-0190.v26i4a168843

Pereira, M. G. L., Vincensi, Y., Alencar, G. P., \& Lima, L. E. M. (2020). Efeitos do treinamento de força no consumo máximo de oxigênio em indivíduos cardiopatas sedentários. Revista Contribuciones a las Ciencias Sociales, Enero, 1-13, 2020. https://www.eumed.net/rev/cccss/2020/01/individuos-cardiopatassedentarios.html

Pícoli, T. S., Figueiredo, L. L., \& Patrizzi, L. J. (2011). Sarcopenia e envelhecimento. Fisioterapia em Movimento, 24(3), 455-462. http://dx.doi.org/10.1590/S0103-51502011000300010

Pillatt, A. P., Nielsson, J., \& Schneider, R. H. (2019). Efeitos do exercício físico em idosos fragilizados: uma revisão sistemática. Fisioterapia e Pesquisa, 26 (2), 210-217. https://doi.org/10.1590/1809-2950/18004826022019

Pinto, R. S., Correa, C. S., Radaelli, R., Cadore, E. L., Brown, L. E., \& Bottaro, M. (2014). Short-term strength training improves muscle quality and functional capacity of elderly women. Age, 36 (1), 365-372. https://doi.org/10.1007/s11357-013-9567-2

Queiroz, C. O., \& Munaro, H. L. R. (2012). Efeitos do treinamento resistido sobre a força muscular e a autopercepção de saúde em idosas. Revista Brasileira de Geriatria e Gerontologia, 15(3), 547-553, 2012. https://doi.org/10.1590/S1809-98232012000300015

Roma, M. F. B., Busse, A. L., Betoni, R. A., Melo, A. C., Kong, J., Santarém, J. M., \& Jacob Filho, W. (2013). Effects of resistance training and aerobic exercise in elderly people concerning physical fitness and ability: a prospective clinical trial. Einstein, 11(2), 153-157. https://doi.org/10.1590/s167945082013000200003

Souza, M. T., Silva, M. D., Carvalho, R. Revisão integrativa: o que é e como fazer. (2010). Einstein, 8(1), 102-106. http://doi.org/10.1590/s167945082010rw1134 
Research, Society and Development, v. 10, n. 12, e81101220131, 2021

(CC BY 4.0) | ISSN 2525-3409 | DOI: http://dx.doi.org/10.33448/rsd-v10i12.20131

Tiggemann, C. L., Dias, C. P., Radaelli, R., Massa, J. C., Bortoluzzi, R., Schoenell, M. C. W., Noll, M., Alberton, C. L., \& Kruel, L. F. M. (2016). Effect of traditional resistance and power training using rated perceived exertion for enhancement of muscle strength, power, and functional performance. Age, 38 (2), 42. https://dx.doi.org/10.1007\%2Fs11357-016-9904-3

Whittemore, R., \& Knafl, K. (2005). The integrative review: updated methodology. Journal of Advanced Nursing, 52 (5), 546-553. https://doi.org/10.1111/j.1365-2648.2005.03621.x

Wójcicki, T. R., \& Mcauley, E. (2014). Manutenção de Intervenções de Atividade Física: premissas para eficácia individual. Revista Família, Ciclos de Vida e Saúde no Contexto Social, 2 (2), 188-193. https://doi.org/10.18554/refacs.v2i2.1171

World Health Organization. (2017). Fact sheet - 10 facts on ageing and health: 1 May 2017. WHO. https://www.who.int/news-room/fact-sheets/detail/10-factson-ageing-and-health

World Health Organization. (2020). Fact Sheet - Physical activity: 26 November 2020. WHO. https://www.who.int/news-room/fact-sheets/detail/physicalactivity 